\title{
Single photon quantum erasing: a demonstration experiment
}

\author{
T L Dimitrova ${ }^{1}$ and A Weis \\ Physics Department, University of Fribourg, Chemin du Musée 3, CH-1700 Fribourg, Switzerland \\ E-mail: antoine.weis@unifr.ch and doradimitrova@uni-plovdiv.bg
}

\begin{abstract}
In the conventional interpretation of quantum mechanics the interference of particles in a two-beam interferometer is closely related to the problem of which-way information. One of the mysteries of quantum mechanics relies on the assumption that the wavefunction of each photon propagates simultaneously along both classically allowed paths, and that interference arises as a consequence of the indistinguishability of those paths. Any attempt to obtain which-way information by putting individual labels on the photons in each pathway inevitably destroys interference. However, even in cases in which the photons carry which-way labels, it is possible to erase those labels after the particle has left the interferometer. The erasing process (partly or completely) destroys the which-way information, and thereby restores interference. This phenomenon is known as quantum erasing. Here we present a lecture demonstration experiment of quantum erasing based on a MachZehnder interferometer operated with single photons.
\end{abstract}

\section{Introduction}

In introductory quantum mechanics, university students are faced with abstract novel concepts such as quantum mechanical states represented by state vectors, state superposition, coherence, interference and entanglement, to name just a few. The students learn basic mathematical rules to transform those entities and to describe their evolution in space and time. From earlier classes on classical physics, students are used to seeing physical phenomena visualized by lecture demonstration experiments or in student laboratories. They often experience difficulties with the abstract world of quantum mechanics, in which demonstration experiments are scarce. Since quantum mechanics is mainly applied to describe the phenomena at the

\footnotetext{
1 Present address: Physics Faculty, University of Plovdiv 'Paisii Hilendarski', Tzar Assen Str. 24, BG-4000 Plovdiv,
} Bulgaria. 

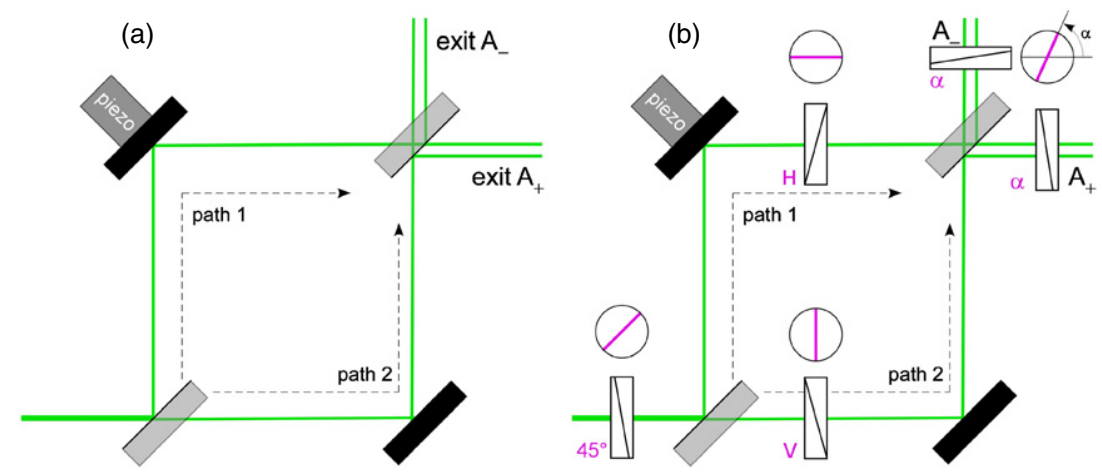

Figure 1. (a) Basic Mach-Zehnder interferometer with piezo-control of the path length difference; (b) modification of the MZI for observing the wave-erasing phenomenon.

atomic and sub-atomic scale, students may erroneously believe that there are no convincing demonstrations of quantum effects in macroscopic setups. Superfluidity and superconductivity are well-known counter-examples that show quantum behaviour at the macroscopic scale. The single photon interference experiments presented here are another beautiful way to discuss macroscopic quantum phenomena. The discussion of quantum interference in Young's double slit experiment is a standard topic in any introductory quantum mechanics lecture. However, due to the small separation of the slits, the spatial separation of the interfering beams is hardly visible by the unaided eye. This difficulty can be circumvented by the use of a two-beam interferometer, such as the Mach-Zehnder interferometer (MZI), which allows realizing tablesize beam separations and interference of quantum states. The specific didactical advantages of such interferometers were addressed before $[1,2]$.

In a recent publication [2] we have described a MZI-based lecture demonstration experiment of single-photon interference which illustrates the wave-particle duality of light by demonstrating the transition from the particle aspect of light to its wave aspect. The experiment also demonstrates in a convincing way that each photon 'interferes with itself' (phrasing of Dirac [3]). In the present paper we describe an extension of the latter apparatus for demonstrating the intriguing phenomenon of single-photon quantum erasing.

\section{Interference of polarized waves and wave erasing}

We start by discussing the interference of classical waves in the two-beam Mach-Zehnder interferometer shown in figure 1(a). A first beamsplitter separates a monochromatic wave of intensity $I_{0}$ into two coherent waves which are recombined by a second beamsplitter after having propagated along different paths. Two pairs of beams emerge from the interferometer at the exit ports $A_{+}$and $A_{-}$. The two beams in each exit port interfere in a constructive or destructive manner depending on the relative phase $\Delta \varphi$ that they have accumulated during their propagation through the interferometer. If the interfering beams propagate along the same direction, the (complementary) intensities emerging at $A_{+}$and $A_{-}$are given by

$$
I_{ \pm}(\Delta \varphi)=\frac{I_{0}}{2}(1 \pm \cos \Delta \varphi) .
$$

The relative phase $\Delta \varphi$ of the interfering beams is determined by the difference of the path lengths they travel, which can be controlled by translating the upper-left mirror with the help of a piezoelectric transducer (PZT). 


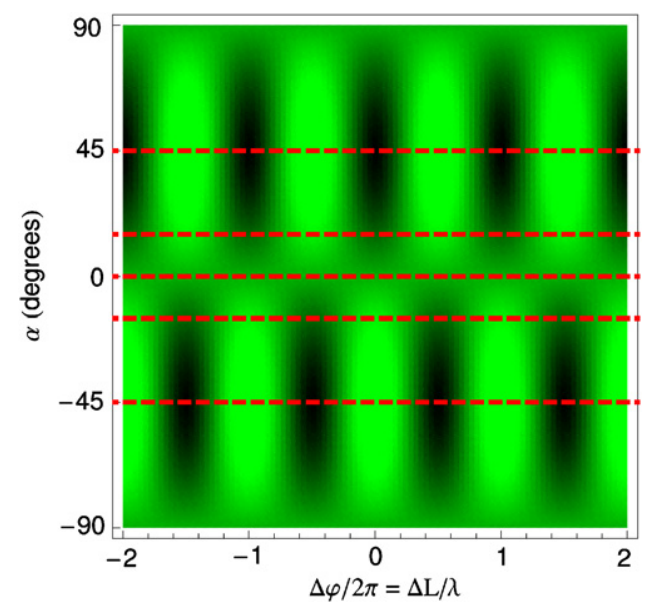

Figure 2. Calculated intensity of the fringe pattern after the eraser as given by (2). The dashed lines refer to the eraser settings of figure 5 .

In practice one adjusts the interferometer such that the interfering beams propagate along slightly different directions, in which case the uniform transverse intensity distribution of the emerging light changes into a system of equidistant bright and dark fringes. The number of fringes is proportional to the angle formed by the interfering beams. The intensity at each position in the fringe pattern varies periodically with $\Delta \varphi$, according to (1) with a positiondependent phase offset. As a consequence the fringe pattern moves uniformly in its transverse direction when $\Delta \varphi$ is varied by applying a linear voltage ramp to the piezo.

If one inserts orthogonal linear polarizers (horizontal and vertical, respectively) in the two paths of the MZI in figure 1(b), the fringe patterns at both exit ports become homogeneous spots, each of intensity $I_{0} / 4$. Since orthogonally polarized beams do not interfere, the spot intensities represent the incoherent sum of the intensities of the individual beams, each beam losing half of its intensity at each beam splitter and at the polarizer. Interference can be restored by the insertion of a third polarizer (eraser) after one of the exits, which yields the fringe pattern (derived in appendix A),

$$
I_{ \pm}(\Delta \varphi, \alpha)=\frac{I_{0}}{8}(1 \pm \sin 2 \alpha \cos \Delta \varphi)
$$

where $\alpha$ is the orientation of the eraser with respect to the horizontal ${ }^{2}$. For reasons that will become evident in the following section the polarizer at the exit is called the 'eraser', and we speak of classical erasing when referring to the restoration of interference by that polarizer. Figure 2 shows a contour plot diagram of equation (2), in which the cuts indicated by the dashed lines correspond to the eraser settings used in figure 5 below.

The erasing phenomenon with classical waves can be readily understood: two orthogonally polarized beams cannot interfere and, hence, produce no fringes. When the erasing polarizer is inserted with an orientation that differs from the horizontal $\left(\alpha=0^{\circ}\right)$ or the

\footnotetext{
2 The factor $I_{0} / 8$ in equation (2) is only valid when the incident light is either unpolarized or polarized at $45^{\circ}$ with respect to the horizontal.
} 
vertical $\left(\alpha= \pm 90^{\circ}\right.$ ), the optical fields of both beams have interfering polarization components along the eraser direction, and can thus interfere. The (signed) fringe visibility $V_{ \pm}$given by

$$
V_{ \pm}=\frac{I_{ \pm}(0, \alpha)-I_{ \pm}(\pi, \alpha)}{I_{ \pm}(0, \alpha)+I_{ \pm}(\pi, \alpha)}= \pm \sin 2 \alpha
$$

becomes maximal for $\alpha= \pm 45^{\circ}$. It is complementary at the two outputs and changes sign for $\alpha \rightarrow-\alpha$.

Hillmer and Kwiat [4] have presented a do-it-yourself realization of the wave-erasing phenomenon using diffraction of an (unattenuated) beam from a laser pointer by a thin wire. When the partial waves passing on either side of the wire are marked by orthogonal polarizers, the diffraction, i.e. interference pattern, disappears. The interference can be restored with maximal contrast when a polarizer oriented at $\pm 45^{\circ}$ is inserted in the far field. Although the experiment is presented with strong light only (wave erasing), [4] contains a valuable discussion of the underlying quantum mechanical aspects (quantum erasing) addressed below.

\section{Single photon interference}

The discussion of the erasing phenomenon at a single particle level (quantum erasing) is more intriguing, since it is related to the quantum mechanical question of which-way information. Let us first consider the case in which a single photon traverses the MZI of figure 1(a). The photon's wavefunction is coherently split by the first beamsplitter and evolves along both classical paths. The wavefunction of the photon inside the interferometer is described by

$$
\left|\Psi_{\text {inside }}\right\rangle=r|1\rangle \mathrm{e}^{\mathrm{i} \Delta \varphi}+t|2\rangle,
$$

where the state vectors $|1\rangle$ and $|2\rangle$ refer to the two paths, and $\Delta \varphi$ is the phase difference introduced above. The factors $r$ and $t$ are the amplitude reflection and transmission coefficients imposed by the first beamsplitter. The second beamsplitter further splits the wavefunction into four components, so that the wavefunction of the photon exiting the interferometer is given by

$$
\left|\Psi_{\text {out }}\right\rangle=r^{2}|-\rangle \mathrm{e}^{\mathrm{i} \Delta \varphi}+t r|+\rangle \mathrm{e}^{\mathrm{i} \Delta \varphi}+t^{2}|-\rangle+r t|+\rangle,
$$

where the state vectors $| \pm\rangle$ refer to the propagation directions in the output ports $A_{ \pm}$. The probabilities $w_{+}$and $w_{-}$for detecting the photon at either $A_{+}$or $A_{-}$are then given by

$$
w_{ \pm}=\left|\left\langle \pm \mid \Psi_{\text {out }}\right\rangle\right|^{2}=\frac{1}{2}(1 \pm \cos \Delta \varphi),
$$

which agree with the intensities (1) derived for wave interference. The amplitude transmission and reflection coefficients are $t=1 / \sqrt{2}$ and $r=\mathrm{i} / \sqrt{2}$, respectively.

The number of photons per second detected at each output is given by $R_{ \pm}=R_{0} w_{ \pm}$, where $R_{0}$ is the rate of photons (number of photons per second) entering the interferometer. The single photon events can be rendered visually (showing pulses from a single photon detector on an oscilloscope) or acoustically (transforming the single photon detector pulses to clicks rendered by a loudspeaker). Quantum interference of individual photons can thus be visualized [2] as a periodic modulation of the single photon event rate (average frequency of pulses or clicks) when $\Delta \varphi$ is varied. The 'weirdness' of single photon interference lies in the fact that each photon behaves differently when both paths are open and when one path is blocked, as discussed in [2].

\section{Quantum erasing}

Single photon interference is a consequence of the indistinguishability of the interfering paths shown in figure 3(a). Any attempt to obtain which-way information leads unavoidably to 

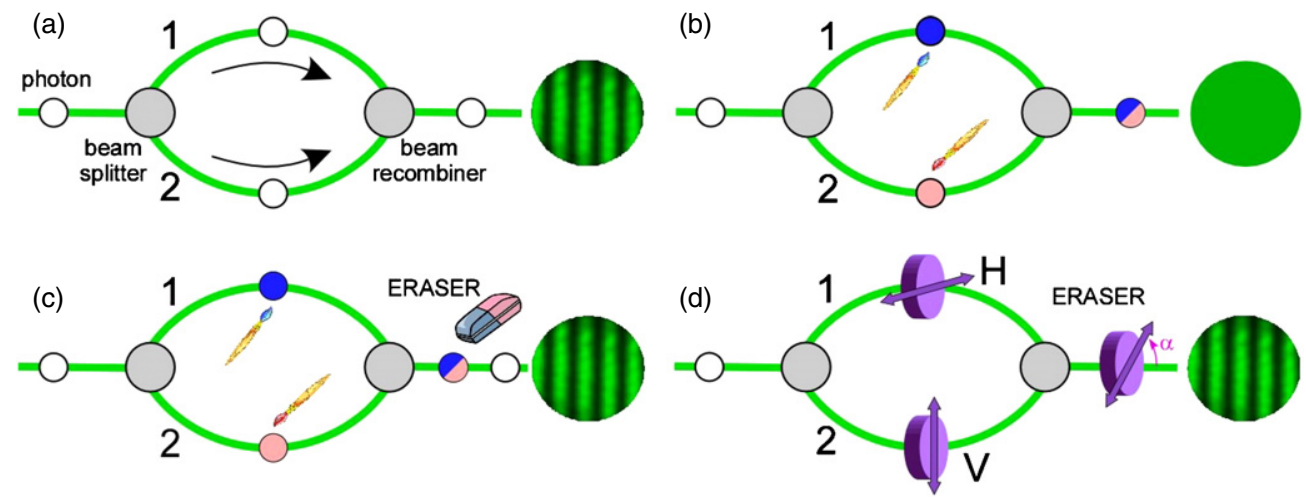

Figure 3. (a) Principle of a two-path interferometer. (b) Destruction of interference by path labelling paintbrushes (top: blue/dark, bottom red/grey). (c) Restoring of interference by erasing the path labels imposed in (b). (d) Practical realization of the labelling and erasing with linear polarizers.

the destruction of interference. Classical gedanken experiments for obtaining which-way information have considered the detection of a 'trace' left by the photon in each path, such as the recoil of one of the path direction changing elements (mirror, double slit) or the interaction of the photon with an atom placed near one of the paths. An alternative way to obtain whichway information after the photon has left the interferometer is the application of path-specific labels on the photons in each path. This is represented in figure 3(b), where the photons in each path are symbolically labelled by a specific colour ${ }^{3}$. By inserting a specific ('blue' or 'red') colour filter into the recombined beam, one can, in principle, gain a posteriori knowledge on the path taken by the photon. As a consequence, no interference will be observed on the projection screen. It is not necessary to actually insert the label-identifying filter to see the interference disappear. The mere fact that which-way information is carried by the photons suffices to destroy interference.

In some circumstances, however, it is possible to wipe out the specific path labels by a suitable device (shown symbolically as a colour eraser in figure 3(c)), so that the photon no longer carries which-way information. In that case interference will be restored with full contrast. In our experiment we have opted for a labelling by mutually orthogonal (horizontal $H$ and vertical $V$ ) linear polarizers (figure 3(d)). The erasing element in that case is a suitably oriented linear polarizer inserted after the interferometer. Since the information erasing is done on individual light quanta, the process is called quantum erasing. The quantum eraser setup is identical to the wave erasing setup shown in figure 1(b) and discussed in section 2.

The quantum mechanical treatment of the single photon quantum-erasing experiment involves the delocalized wavefunction of the photon. As shown in appendix $\mathrm{B}$, the wavefunction of the photon in the interferometer after having passed the $H$ and $V$ polarizers is given by

$$
\left|\Psi_{\text {after } H / V}\right\rangle=\frac{1}{\sqrt{2}}\left(r|1\rangle|H\rangle \mathrm{e}^{\mathrm{i} \Delta \varphi}+t|2\rangle|V\rangle\right) .
$$

This wavefunction represents a single particle entangled state in which the photon's internal degree of freedom (polarization) is entangled with its external degree of freedom (direction

\footnotetext{
3 The labels 'red' and 'blue' chosen here are to be understood as symbolic labels. However, one might think of a quantum eraser variant in which the light frequencies in both arms are shifted to the blue and red, respectively (for instance by acousto-optic modulators), and where a tunable mixer would serve as eraser.
} 


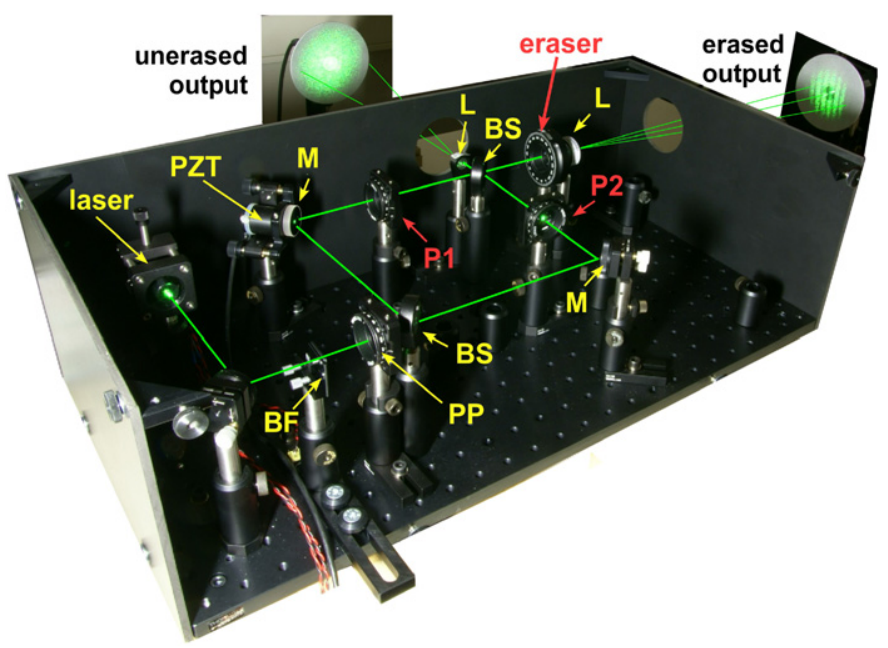

Figure 4. Mach-Zehnder interferometer for the demonstration of erasing with waves and with single photons (quantum erasing). Symbols are explained in the text. The green lines are drawn by hand to illustrate the light paths.

of propagation). As shown in appendix B, the eraser action on this state after it has left the interferometer yields the photon detection probabilities

$$
w_{ \pm}=\frac{1}{8}(1 \pm \cos \Delta \varphi \sin 2 \alpha),
$$

which are identical to the corresponding expression (2) for the intensities of classical waves.

We draw the reader's attention to further useful discussions $[4,5]$ of the relation between quantum interference and which-way measurements.

\section{Experimental setup}

Figure 4 shows a photograph of the Mach-Zehnder interferometer that we use to demonstrate quantum erasing. It is similar to the apparatus that we used previously to demonstrate the wave-particle duality [2]. The beam from a green laser module $(\lambda=532 \mathrm{~nm}, 5 \mathrm{~mW})$ is directed to the interferometer. Prior to entering the interferometer, the intensity of the laser beam can be attenuated by an insertable blocking filter (BF) with an optical density of 4-5. A variable filter (not shown in the photograph) placed after BF allows a smooth variation of the light intensity by a factor of 100 .

The particular laser used for the experiment emits two modes (intensity ratio 5:1) spaced by typically $30 \mathrm{GHz}$, giving the beam an overall coherence length of $1 \mathrm{~cm}$, more than the path length difference $\Delta L$ of the two interfering beams. The coherence length associated with each mode was approximately $40 \mathrm{~cm}$, which corresponds to the MZI dimensions. The quantum interference experiments can be performed under conditions where the average spatial separation of consecutive photons is larger than the individual photon's coherence length, thereby assuring that there is, at each moment, only a single photon in the apparatus.

The Mach-Zehnder interferometer consists of two (non-polarizing) 50/50 beam splitters (BS) and two mirrors (M). One of the mirrors is mounted on a PZT by which the path length difference $\Delta L$ of the two interfering beams, and hence $\Delta \varphi$, can be varied. At the 
exits of the MZI, the interfering beams are expanded by lenses (L) and projected onto metal discs for visualizing the interference patterns. Both projection screens have small central apertures, behind which a photodiode and a photomultiplier are mounted, respectively. The photomultiplier is equipped with two interference filters and a set of narrow collimating apertures that suppress ambient light at a sufficiently low level for permitting demonstrations with single photons in a fully lit environment [2]. The labelling polarizers (P1 and P2) are mounted in rotation stages and are oriented horizontally and vertically, respectively. In order to insure a maximal contrast, a prepolarizer (PP) oriented at $45^{\circ}$ with respect to the horizontal is inserted before the MZI. The erasing polarizer (eraser), also mounted on a rotation stage, can be inserted at one of the exit ports of the MZI.

The MZI can be operated in two modes. With strong light we use projection on the screens for demonstrations to small groups of students, or wall projection for large auditorium presentations. In the scanning mode of operation we display the signals of the photomultiplier (or photodiode) on an oscilloscope while scanning the path length difference through a linear voltage ramp applied to the PZT. In an auditorium the oscilloscope traces can be shown by means of a multimedia projector.

\section{Demonstration experiments}

Prior to demonstrating the quantum-erasing phenomenon, it is necessary to present the main features of Mach-Zehnder interferometry with waves and photons introduced above. For this purpose one removes the $\mathrm{BF}$, the polarizers $\mathrm{P} 1, \mathrm{P} 2$ and the eraser. Once the students are acquainted with the projection and scanning modes, the quantum-erasing demonstration can be shown. When inserting the (pre-aligned) orthogonal polarizers P1 and P2 the projected patterns are seen as light spots with a uniform intensity distribution which show no interference fringes. The lost interference is recovered when the eraser is inserted after one of the exit ports of the MZI. First, wave erasing with strong light is demonstrated. When the eraser is pre-oriented to $\alpha=+45^{\circ}$ or $\alpha=-45^{\circ}$ the projected fringe pattern reappears with maximal contrast. The photograph in figure 4 shows simultaneously the uniform pattern of the unerased exit port (centre, top) and of the erased beam (right). The degree of erasing can be varied by changing the eraser orientation. Typical results are shown in figure 5 both in projection mode and in scanning mode. The fringe contrast is seen to vary smoothly as the eraser orientation $\alpha$, measured with respect to the horizontal plane, is varied. Interference disappears for the trivial cases of $\alpha=0^{\circ}$ (and $\alpha=90^{\circ}$, not shown) and becomes maximal for $\alpha= \pm 45^{\circ}$. The contrast variation obeys the $\alpha$-dependence given by (1). In particular, the inversion of dark and bright fringes when going from $\alpha$ to $-\alpha$ (figure 2) can be observed in figure 5. The slight shift of the fringes in the series is due to thermal drifts of the interferometer during recordings with different settings of $\alpha$.

After insertion of the BF quantum erasing can be shown to occur in single photon events. The experiments are performed in the scanning mode, and the results are shown in columns 3 and 4 of figure 5 . The interferograms in column 3 represent recordings of single time traces, in which the pulses produced by individual photons can be distinguished. In the cases of maximum contrast $\left(\alpha= \pm 45^{\circ}\right)$ one clearly recognizes a modulation of the photon pulse density that is correlated with the strong light fringe patterns of columns 1 and 2 . The contrast of the recordings for $\alpha= \pm 15^{\circ}$ is weaker and the signal modulation is difficult to see in the single-shot time traces. However, when several scans are averaged with the help of a digital oscilloscope (column 4), one clearly observes the reduced contrast for $\alpha= \pm 15^{\circ}$, and the absence of fringes for $\alpha=0^{\circ}$. 

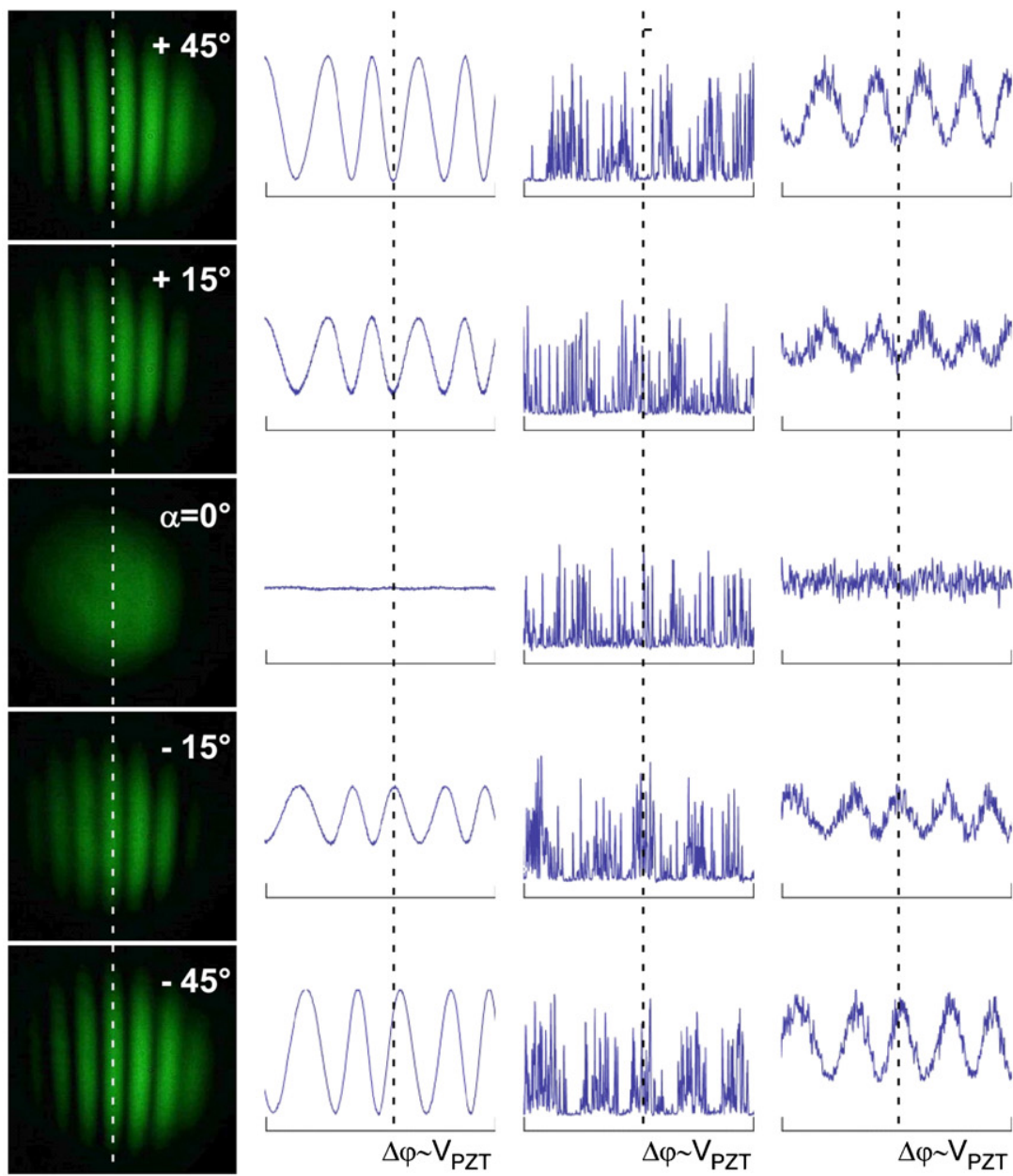

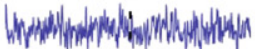
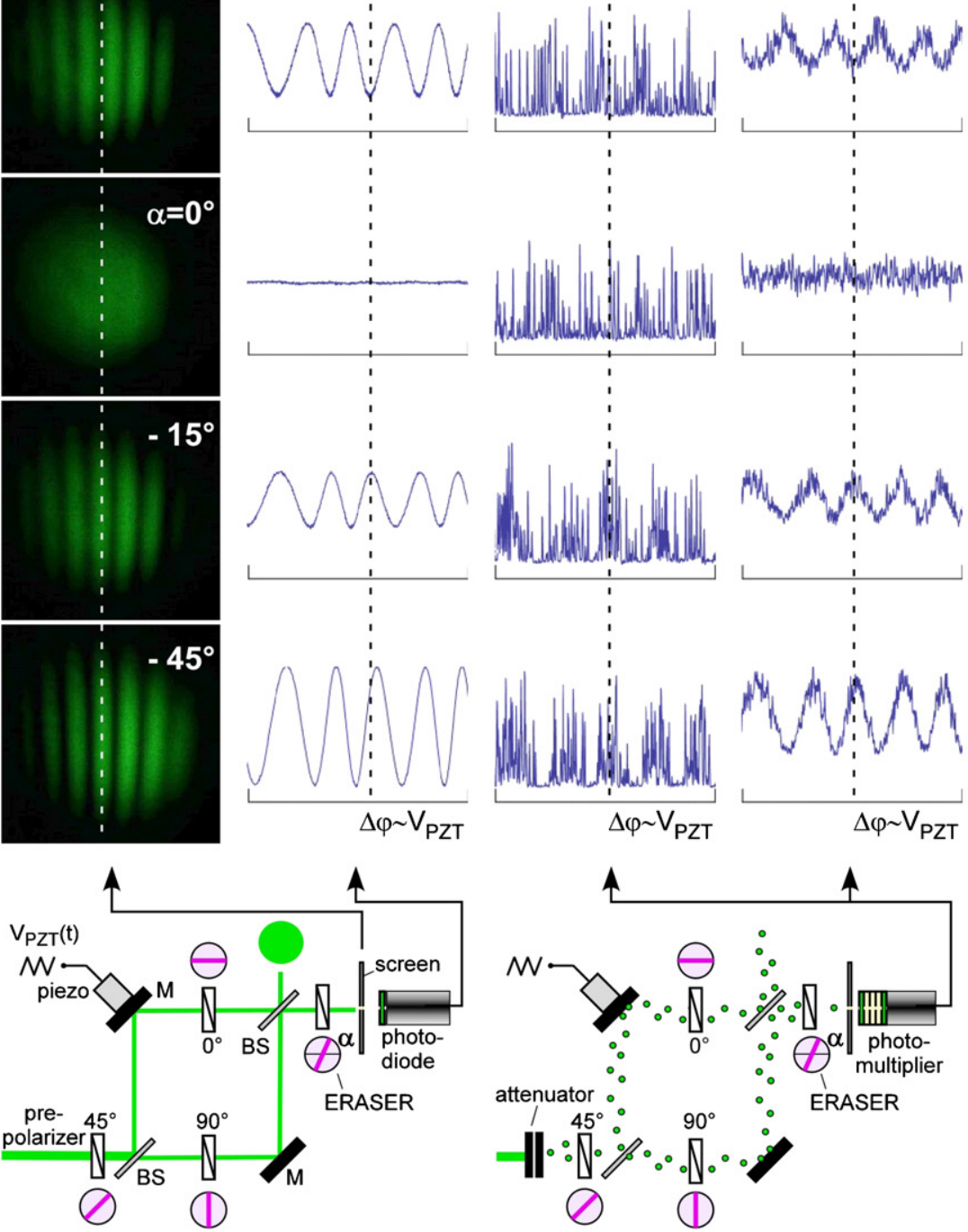

Figure 5. Demonstration of erasing with waves (columns 1,2) and with single photons (columns 3 , 4). Top to bottom the eraser orientation is varied and the values of $\alpha$, with respect to the horizontal, are given as inserts in the photographs.

Columns 1 and 2 show the erasing phenomenon with classical waves, while column 3 shows the phenomenon of quantum erasing with single photons. The time-averaged data in column 4 represent an intermediate case, in which both the wave aspect (sinusoidal modulation) and the particle aspect (granularity of the signal) of erasing are visible. 
A similar quantum-erasing experiment using a MZI was presented by Schneider and LaPuma [5]. The experiment used a fixed path length difference $\Delta L$ and low light level signals were recorded with a consumer-grade CCD camera. In that experiment the average photon number in the interferometer was $10^{-2}$. However, the CCD camera did not permit them to show the results on a photon-by-photon basis. In our experiment, the trivial, albeit powerful use of the piezo-driven mirror, together with photomultiplier detection permits the demonstration of quantum erasing with single particle detection via the rapid scanning between constructive and destructive interference.

\section{Summary and discussion}

We have presented a lecture demonstration experiment for the phenomenon of quantum erasing based on two-path interference in a Mach-Zehnder interferometer. A preliminary account of the present results was presented in [6]. When which-way labelling information is imposed by orthogonal linear polarizers in the two paths, interference disappears at both interferometer exits. Interference with a very high visibility is recovered when the exiting light is made to pass an erasing polarizer oriented along the bisectrix of the labelling polarizers.

The apparatus allows a demonstration of several features of the erasing process both with strong light and with single photons. While the erasing phenomenon is readily understood when the light is treated as a classical wave, a lecture demonstration of quantum erasing with single photons is well suited to introduce and discuss various aspects of quantum mechanics, such as the wave-particle duality, since the apparatus produces a real-time demonstration of the gradual build-up of a smooth (wave) interference pattern from superposed single photon events. The apparatus is also well suited-and probably represents the simplest way-to introduce the fundamental concept of (single particle) entanglement, described by (7).

As discussed by Kwiat and Englert [7] the role of the erasing polarizer is to partition the photons from the MZI in two subsets: transmitted photons and blocked photons. For any two orthogonal orientations ( $\alpha, \alpha+\pi / 2)$ of the eraser at a given exit port, the resulting fringe patterns are therefore shifted by half a period. This can also be seen from (2) and (8), which imply that $I_{ \pm}(\Delta \varphi, \alpha+\pi / 2)=I_{\mp}(\Delta \varphi, \alpha)$. The patterns are thus complementary and the sum of their intensity distributions produces patterns with no fringes. A similar property is seen when comparing the fringe patterns at both exit ports after erasers with identical orientations. Here too the patterns are complementary, as implied by the \pm sign in (2) and (8), and the fringes add to a homogeneous pattern.

The dual nature of light is reflected in the fact that perfect fringe visibility (wave nature) and full which-way information (particle nature) are mutually exclusive. The former is obtained for $\alpha=+45^{\circ}$ and $\alpha=-45^{\circ}$, while the latter occurs for $\alpha=0^{\circ}$ or $90^{\circ}$. Any eraser orientation that differs from those four values relaxes on both aspects, and it was shown (e.g. by Englert [8]) that the degree of fringe visibility and the degree of which-way information obey an inequality. The visibility of the fully erased single photon interferograms (rightmost traces for $\alpha= \pm 45^{\circ}$ in figure 5) does not reach $100 \%$ because of background photons and interferometer drifts during signal averaging. Improving our apparatus from a demonstration device to a research grade device should allow a quantitative study [8] of the wave-particle duality as reported by Schwindt and co-workers [9].

One of the strange aspects of quantum erasing is the fact that the choice (via the orientation of the eraser) of whether one wants to observe wave-like properties (via interference) or particle-like properties (via which-way information) can be taken after the photon has left the interferometer. Unfortunately, the random time distribution of individual photons does not allow one to know the instant at which the photon leaves (enters) the interferometer. However, 
by using a sufficiently fast polarization switching device at a sufficiently large distance after the MZI, one can always ascertain that the photon will have left the interferometer at the moment of decision taking. For this one would only count photons during a sufficiently short time interval following the setting of the eraser. This timing difficulty can also be circumvented in more elaborate experiments that use entangled photon pairs produced by parametric downconversion (as discussed, e.g., by Herzog et al [10]). By measuring photon correlations it is then even possible to decide whether to read out or to erase the which-way information after the actual detection of the photon [11].

The concept of erasing which-path information was coined by Scully and Drühl [12] in 1982 and quantum erasing has since been studied in many experiments using mainly entangled photon pairs ${ }^{4}$. The experiment presented here shows quantum erasing in its simplest formusing single photon entangled states - in a way that is adapted to lecture hall demonstrations.

\section{Acknowledgments}

The authors thank Dr Natascia Castagna for her critical reading of the manuscript and checking the maths. One of us (TLD) acknowledges financial support from the Physics Department and the Fondation du Fonds de la Recherche of the University of Fribourg.

\section{Appendix A. Derivation of equation (2)}

The wave erasing expression in equation (2) can be most easily derived using the Jones vector formalism, in which two component vectors (spinors) $\Psi$ describe the state of polarization of the light. The basis vectors are chosen to be the horizontally and vertically polarized waves. The optical field of the laser beam after the prepolarizer oriented at $45^{\circ}$ is given by

$$
\Psi_{0}=\frac{E_{0}}{\sqrt{2}}\left(\begin{array}{l}
1 \\
1
\end{array}\right)
$$

The BS split each incident beam into a reflected and a transmitted beam of equal intensity. The effect of an ideal beam splitter is described by multiplying the incident spinor by the amplitude reflection and transmission factors [14]:

$$
r=\frac{\mathrm{i}}{\sqrt{2}} \quad \text { and } \quad t=\frac{1}{\sqrt{2}},
$$

which follow from the Fresnel laws. Figure A1 shows the amplitudes of the optical fields at different positions along the possible paths 1 and 2 .

The horizontal and vertical polarizers are described by the matrices

$$
M_{H}=\left(\begin{array}{ll}
1 & 0 \\
0 & 0
\end{array}\right) \quad \text { and } \quad M_{V}=\left(\begin{array}{ll}
0 & 0 \\
0 & 1
\end{array}\right)
$$

respectively. The erasing polarizer is oriented at an angle $\alpha$ with respect to the horizontal, so that its matrix is given by

where

$$
M_{\alpha}=R^{-1}(\alpha) M_{H} R(\alpha)=\left(\begin{array}{cc}
\cos ^{2} \alpha & \sin \alpha \cos \alpha \\
\sin \alpha \cos \alpha & \sin ^{2} \alpha
\end{array}\right),
$$

$$
R(\alpha)=\left(\begin{array}{cc}
\cos \alpha & \sin \alpha \\
-\sin \alpha & \cos \alpha
\end{array}\right)
$$

is the rotation matrix in two dimensions.

4 A discussion of various undergraduate experiments using entangled photon pairs is given, e.g., by Galvez et al [13]. 


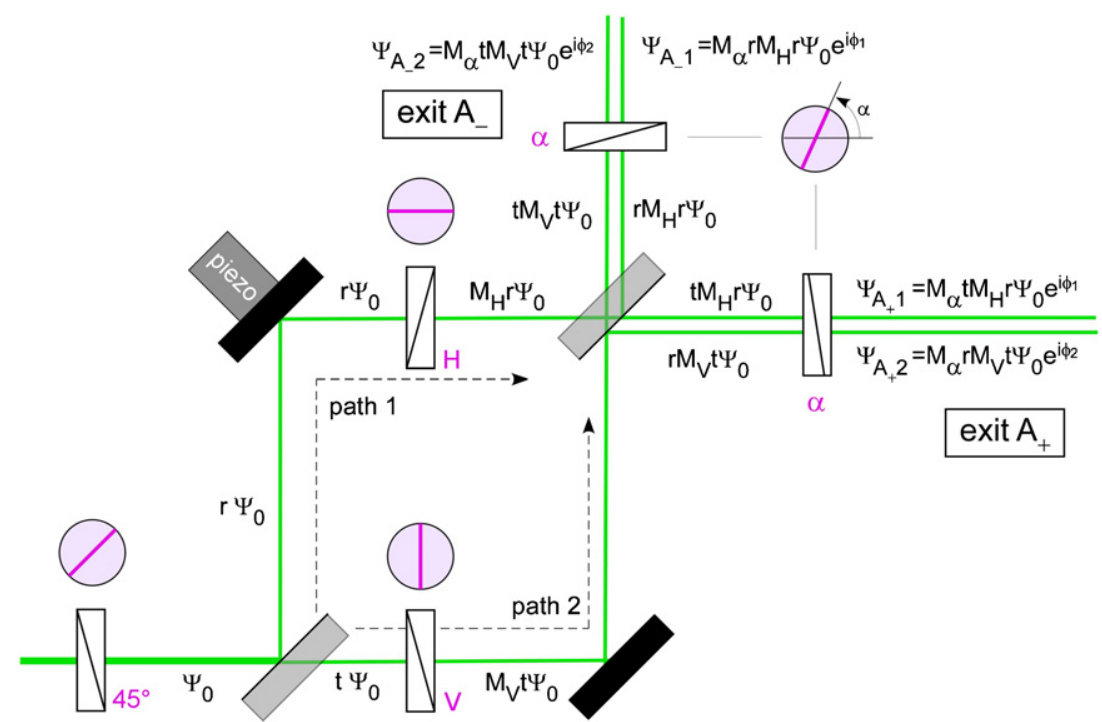

Figure A1. The field amplitudes of the various beams in the quantum erasing setup. The spatial phases acquired by the individual beams are only given for the expressions of the four fields at the exits.

Each beam in the interferometer acquires a spatial phase $\mathrm{e}^{\mathrm{i} \varphi_{\mathrm{i}}}$ with

$$
\varphi_{1}=2 \pi \frac{L_{1}}{\lambda}+\delta \varphi \quad \text { and } \quad \varphi_{2}=2 \pi \frac{L_{2}}{\lambda},
$$

where $L_{i}$ are the lengths of the paths $(i=1,2)$ travelled by each beam, and

$$
\delta \varphi=2 \pi \frac{\Delta L}{\lambda}=\beta V
$$

is the additional (voltage controlled) phase shift due to the piezo-controlled displacement of the mirror, $V$ being the voltage applied to the piezo. In order to simplify the mathematical expressions in figure A1, we have omitted those phase factors and write them only in the final expressions at the exit ports.

The intensity $I_{-}$at the (erased) exit $A_{-}$is given by squaring the sum of the amplitudes shown in figure 5 , which yields, after some algebra

$$
I_{-}=\left|\Psi_{A_{-} 1}+\Psi_{A_{-} 2}\right|^{2}=\frac{I_{0}}{8}(1-\cos \Delta \varphi \sin 2 \alpha),
$$

where

$$
\Delta \varphi=2 \pi \frac{L_{1}-L_{2}}{\lambda}+\delta \varphi \equiv \Delta \varphi_{0}+\beta V
$$

Changing the voltage $V$ by a linear ramp thus yields an oscillatory variation of the intensity whose amplitude depends on the orientation $\alpha$ of the eraser.

In a similar way one finds the intensity at the output $A_{+}$to be given by

$$
I_{+}=\left|\Psi_{A_{+} 1}+\Psi_{A_{+} 2}\right|^{2}=\frac{I_{0}}{8}(1+\cos \Delta \varphi \sin 2 \alpha) .
$$

The complementarity of the two output intensities implies that the total intensity

$$
I=I_{+}+I_{B}=\frac{1}{4}
$$

is independent of $\Delta \varphi$. 


\section{Appendix B. Derivation of equations (7) and (8)}

In the case of single photon erasing we use the Dirac bra and ket notation to denote the quantum mechanical state of the system. The state after the prepolarizer is given by

$$
\left|\Psi_{0}\right\rangle=\frac{1}{\sqrt{2}}(|H\rangle+|V\rangle)|i n\rangle
$$

where $|H\rangle$ and $|V\rangle$ denote horizontally and vertically polarized states, and $|i n\rangle$ refers to the propagation direction of the incoming photon. The action of the first beamsplitter is described by the projection operator

$$
P_{B S 1}=r|1\rangle\langle i n|+t| 2\rangle\langle i n| .
$$

where $|1\rangle$ and $|2\rangle$ refer to the propagation directions of the reflected and transmitted beams; $r$ and $t$ are the reflection and transmission coefficients introduced in appendix A. The wavefunction of the photon after the first beam splitter is then given by

$$
\left|\Psi_{B S 1}\right\rangle=P_{B S 1}\left|\Psi_{0}\right\rangle=\frac{1}{\sqrt{2}}(r|1\rangle|H\rangle+t|2\rangle|V\rangle+r|1\rangle|V\rangle+t|2\rangle|H\rangle),
$$

Introducing the spatial phase difference $\Delta \varphi$ acquired by the beams we obtain the wavefunction before the $H$ and $V$ polarizers:

$$
\left|\Psi_{\text {before } H / V}\right\rangle=\frac{1}{\sqrt{2}}\left(r|1\rangle|H\rangle \mathrm{e}^{\mathrm{i} \Delta \varphi}+r|1\rangle|V\rangle \mathrm{e}^{\mathrm{i} \Delta \varphi}+t|2\rangle|H\rangle+t|2\rangle|V\rangle\right) .
$$

The $H$ polarizer in beam 1 and the $V$ polarizer in beam 2 pass only the corresponding polarization states, and the action of the polarizers is therefore described by the projection operator

$$
P_{H / V}=|H\rangle\langle H|| 1\rangle\langle 1|+| V\rangle\langle V|| 2\rangle\langle 2| .
$$

After the polarizers the (intra-interferometer) state vector is reduced to

$$
\left|\Psi_{\text {after } H / V}\right\rangle=P_{H / V}\left|\Psi_{\text {before } H / V}\right\rangle=\frac{1}{\sqrt{2}}\left(r|1\rangle|H\rangle \mathrm{e}^{\mathrm{i} \Delta \varphi}+t|2\rangle|V\rangle\right) \text {. }
$$

The second beam splitter splits each subcomponent of the wavefunction (B.6) into reflected and transmitted parts yielding outputs along the directions $|+\rangle$ and $|-\rangle$. Its action is thus described by the projection operator

$$
P_{B S 2}=r|-\rangle\langle 1|+t|+\rangle\langle 1|+r|+\rangle\langle 2|+t|-\rangle\langle 2| \text {. }
$$

The wavefunction of the photon that has left the interferometer, but has not yet passed the eraser is then given by

$$
\begin{aligned}
\left|\Psi_{\text {before eraser }}\right\rangle & =P_{B S 2}\left|\Psi_{\text {after } H / V}\right\rangle \\
& =\frac{1}{\sqrt{2}}\left(r^{2}|H\rangle|-\rangle \mathrm{e}^{\mathrm{i} \Delta \varphi}+t r|H\rangle|+\rangle \mathrm{e}^{\mathrm{i} \Delta \varphi}+r t|V\rangle|+\rangle+t^{2}|V\rangle|-\rangle\right) .
\end{aligned}
$$

Let us now consider only the part of the wavefunction that appears at the exit port $A_{-}$, i.e. which propagates along the $|-\rangle$direction. It is obtained by applying the projector $P_{-}=|-\rangle\langle-|$on the last expression, yielding

$$
\begin{aligned}
\left|\Psi_{-}\right\rangle=P_{-}\left|\Psi_{\text {before eraser }}\right\rangle & =\frac{1}{\sqrt{2}}\left(r^{2}|H\rangle|-\rangle \mathrm{e}^{\mathrm{i} \Delta \varphi}+t^{2}|V\rangle|-\rangle\right) \\
& =\frac{1}{2 \sqrt{2}}\left[-|H\rangle \mathrm{e}^{\mathrm{i} \Delta \varphi}+|V\rangle\right]|-\rangle .
\end{aligned}
$$

Let the eraser after the output $A_{-}$be oriented at $\alpha$ with respect to the horizontal. It passes only the state

$$
\left|\Psi_{\alpha}\right\rangle=\cos \alpha|H\rangle|-\rangle+\sin \alpha|V\rangle|-\rangle,
$$

so that its action is described by the projection operator

$$
\begin{aligned}
P_{\alpha} & =\left|\Psi_{\alpha}\right\rangle\left\langle\Psi_{\alpha}\right| \\
& =\left[\cos ^{2} \alpha|H\rangle\left\langle H\left|+\sin ^{2} \alpha\right| V\right\rangle\langle V|+\sin \alpha \cos \alpha(|H\rangle\langle V|+| V\rangle\langle H|)\right]|-\rangle\langle-| .
\end{aligned}
$$


The wavefunction after the eraser is then given by

$$
\begin{aligned}
\left|\Psi_{\text {after eraser }}\right\rangle & =P_{\alpha}\left|\Psi_{-}\right\rangle \\
& =\frac{1}{2 \sqrt{2}}\left[-\cos ^{2} \alpha|H\rangle \mathrm{e}^{\mathrm{i} \Delta \varphi}+\sin ^{2} \alpha|V\rangle+\sin \alpha \cos \alpha\left(|H\rangle-|V\rangle \mathrm{e}^{\mathrm{i} \Delta \varphi}\right)\right]|-\rangle
\end{aligned}
$$

and the probability of detecting the photon after the eraser at the output $A_{-}$is obtained from

$$
w_{-}=\left\langle\Psi_{\text {after eraser }} \mid \Psi_{\text {after eraser }}\right\rangle,
$$

which, after some algebra, yields

$$
w_{-}=\frac{1}{8}(1-\cos \Delta \varphi \sin 2 \alpha),
$$

i.e. the same result as (A.8) obtained in the wave-erasing calculation. The probability for the photon to be detected at the other output is found to be

$$
w_{+}=\frac{1}{8}(1+\cos \Delta \varphi \sin 2 \alpha) \text {. }
$$

\section{References}

[1] Scarani V and Suarez A 1998 Introducing quantum mechanics: one particle interference Am. J. Phys. 66 718-21

[2] Dimitrova T L and Weis A 2008 The wave-particle duality of light: a demonstration experiment Am. J. Phys. 76 137-42

[3] Dirac P A 1982 The Principles of Quantum Mechanics 4th edn (Oxford: Oxford University Press)

[4] Hillmer R and Kwiat P 2007 A do-it-yourself quantum eraser Sci. Am. 296 90-5

[5] Schneider M B and LaPuma I A 2002 A simple experiment of quantum interference and which-way measurement Am. J. Phys. 70 266-71

[6] Dimitrova T L and Weis A 2009 Lecture demonstrations of interference and quantum erasing with single photons Phys. Scr. T 135014003

[7] Kwiat P G and Englert B G 2004 Quantum erasing the nature of reality, or perhaps, the reality of nature? Science and Ultimate Reality: Quantum Theory, Cosmology, and Complexity ed J D Barrow, P C W Davies and C L Harper Jr (Cambridge: Cambridge University Press)

[8] Englert B G 1996 Fringe visibility and which-way information: an inequality Phys. Rev. Lett. 77 2154-7

[9] Schwindt P P D, Kwiat P G and Englert B G 1999 Quantitative wave-particle duality and nonerasing quantum erasure Phys. Rev. A 60 4285-9

[10] Herzog T J, Kwiat P G, Weinfurter H and Zeilinger A 1995 Complementarity and the quantum eraser Phys. Rev. Lett. 17 3034-7

[11] Walborn S P, Terra Cunha M O, Pádua S and Monken C H 2002 A double-slit quantum eraser Phys. Rev. A $650338181-8$

[12] Scully M O and Drühl K 1982 Quantum eraser-A proposed photon correlation experiment concerning observation and 'delayed choice' in quantum mechanics Phys. Rev. A 25 2208-13

[13] Galvez E J, Holbrow C H, Pysher M J, Martin J W, Courtemanche N, Heilig L and Spencer J 2005 Interference with correlated photons: five quantum mechanics experiments for undergraduates Am. J. Phys. 73 127-40

[14] Degiorgio V 1980 Phase shift between the transmitted and the reflected optical fields of a semireflecting lossless mirror is $\pi / 2$ Am. J. Phys. 48 81-2 